\title{
Observed Evolution of the Nebulae and Central Stars
}

\author{
Romuald Tylenda \\ Department for Astrophysics, N. Copernicus Astronomical Centre, \\ Rabiańska 8, 87-100 Toruń, Poland
}

\begin{abstract}
Several points involved in observational studies of evolution of planetary nebulae and their central stars are discussed. It concerns observed masses of nebulae and central stars, nature of $\mathrm{H}$-deficient central stars, role of time-dependent ionization in the nebular haloes, and a recent discovery of strong enrichements in some planetary nebulae.
\end{abstract}

\section{Introduction}

The subject of observed evolution of planetary nebulae (PNe) and their nuclei $(\mathrm{PNn})$ is very large. In fact most of the papers in this volume concern it. It is impossible to cover the whole area within limits of a single talk. Therefore this paper concentrates on some selected topics which, in author's feeling, are important and interesting, but raising problems and questions. The first topic concerns the observed PN mass and its evolution and a danger of getting spurious observational results in this subject. Next, the problem of the observed PNn mass will be discussed where results from different sources do not lead to consistent conclusions. The problem of $\mathrm{H}$-deficient $\mathrm{PNn}$ will also be reviewed briefly. The conclusion is that the main evolutionary path leading to origin of $\mathrm{H}$-deficient $\mathrm{PNn}$, at least of [WC] type, is the last AGB thermal pulse. The nature and origin of the PN haloes has recently received much of interest. We point out on a role of time-dependent effects in these $\mathrm{PN}$ regions which can provide information on recent PNn evolution. Finally, the subject of strong inhomogeneities in element abundances in the PNe will be discussed. This is a very recent problem, although, principal observational facts have been known for decades but misinterpreted or ignored.

\section{Observed evolution of the nebular mass}

One of the important points in the evolution of PNe concerns the observed nebular mass and its evolution. Theoretical considerations show that the PN mass should, in general, increase with time (e.g. Schmidt-Voigt \& Köppen 1987; Marten \& Schönberner 1991). In young PNe it increases because the ionized (i.e. observable) part of the PN increases due to the increasing ionizing flux from the PNn and the expansion of the PN. In old PNe the mass should also increases since the faster expanding PN accretes mass from a slow AGB wind.

At first sight this prediction seems to be well supported by observations. It has been argued in numerous studies that there is a strong positive correlation 


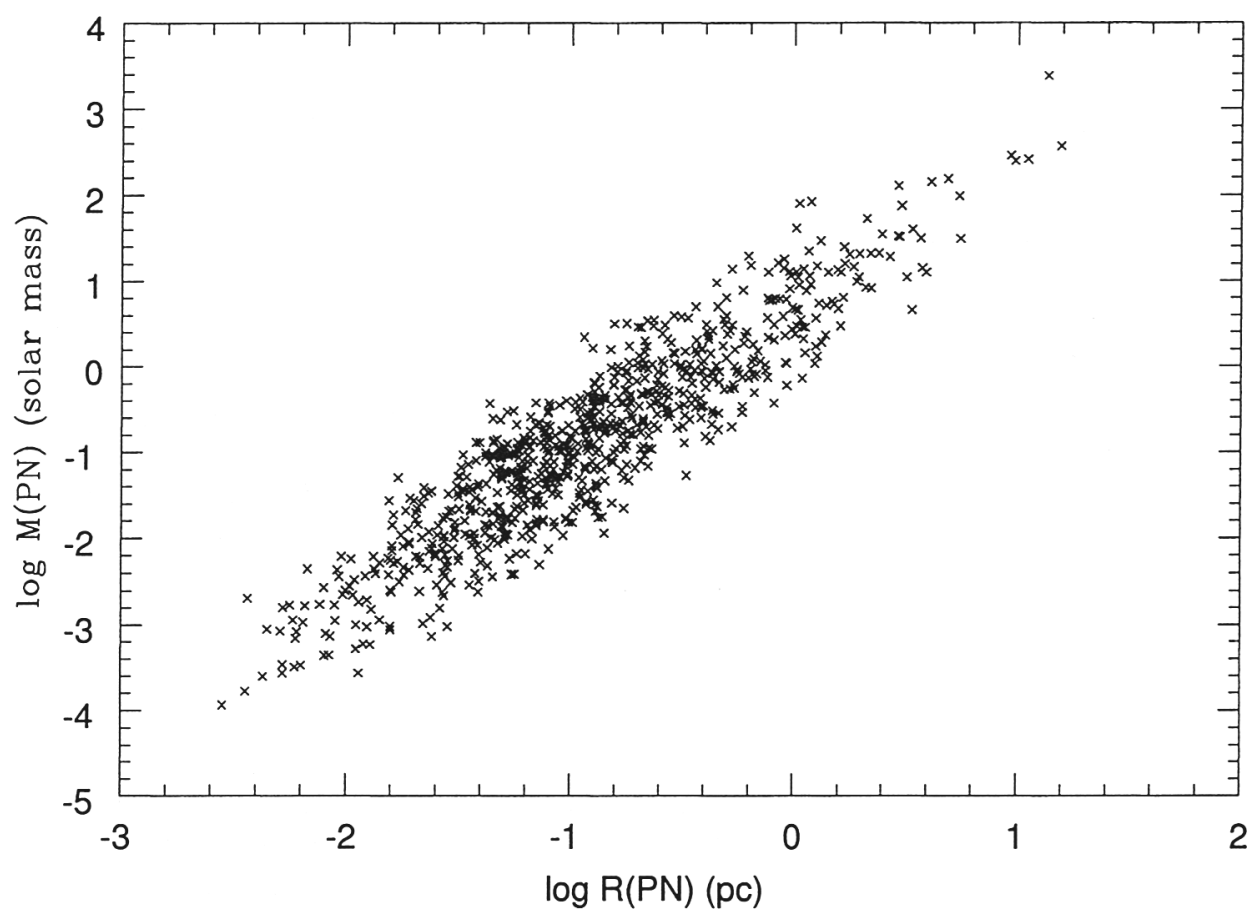

Figure 1. The spurious PN mass-radius relation (see text for details).

between the observed PN mass and the PN radius (e.g. Gathier 1987; Pottasch \& Acker 1989; Pottasch 1992; Bensby \& Lundström 2001).

It is, however, necessary to point out that the observed mass-radius relation, usually found to be remarkably tight, can be largely spurious. It may be an artifact of the method used for observational determination of the PN mass and radius. What is usually plotted in diagrams of this kind is a quantity proportional to $F^{0.5} \theta^{1.5} d^{2.5}$ against $\theta d$ (here $F$ is the nebular flux in $\mathrm{H} \beta$ or radio continuum, $\theta$ is the angular nebular radius, $d$ is the distance). As the flux enters in a small power, in fact, one plots $\theta^{1.5} d^{2.5}$ versus $\theta d$. Thus there is no surprise that the resultant relation appears very tight. The above point has been discussed in Stasińska et al. (1991). An example illustrating the problem is given in Fig. 1. It plots the PN mass against the PN radius, derived as discussed above, for Galactic PNe (over 800 objects) having measured the $\mathrm{H} \beta$ flux and the angular radius (taken from an updated data base of Acker et al. 1992). However, the distances used in the diagram have been randomly selected between 1 and $10 \mathrm{kpc}$. Fig. 1 shows a very nice mass-radius relation, very similar to those usually found, in spite of the fact that the values of distances used are certainly wrong.

To be clear: the above discussion must not be interpreted as a claim that there is no correlation between the PN mass and the PN radius. It only points out that the diagrams of the sort presented in Fig. 1 cannot be used as evidence for a real mass-radius relation. It is practically impossible to say to what extent 
the obtained relation reflects the real relation and to what extent it is due to the observational method itself.

\section{Observed evolution and masses of the central stars}

Theoretical principles of the stellar evolution in the PN phase have been established in considerable detail (see e.g. Blöcker, these proceedings). There is a general consensus that they satifactorily explain main observational aspects of the PNn population. More detailed comparisons between PNn models and the observations often suffer from the distance problem. In particular, this concerns the HR diagram where also uncertainities in observational determining the PNn effective temperature are important.

Hence several attempts investigating distance independent diagrams and using parameters which can be obtained directly (i.e. without assumptions introducing uncertainities) form observations (e.g. Tylenda \& Stasińska 1989; Zhang \& Kwok 1993; Górny et al. 1997). These methods, however, usually have to rely on a particular model of the PN evolution. Therefore the results from them should be considered in statistical terms rather. Drawing conclusions concerning individual objects should be done with great caution.

One of the most important information that can be obtained from comparisons between theoretical PNn models and observations concerns the PNn masses. A general conclusions arising from most of the above studies is that the typical PNn mass is very close to $0.60 \mathrm{M}_{\odot}$. Differences concern details of the PNn mass distribution, in particular, the importance of the high mass tail.

An analysis similar to that in Górny et al. (1997) and Stasińska et al. (1997) but done for an updated observational data base for 182 Galactic PNe gives the following result: median PNn mass is $0.597 \mathrm{M}_{\odot}$, mean $\mathrm{PNn}$ mass is $0.619 \mathrm{M}_{\odot}$ with a standard deviation of $0.054 \mathrm{M}_{\odot}$, the high mass tail $\left(\mathrm{M}_{\mathrm{PNn}}>0.65 \mathrm{M}_{\odot}\right)$ contains $13.2 \%$ of the sample. These numbers, as well as, the mass histogram are not significantly different from those in Stasińska et al. The above result has been obtained adopting the PN mass of $0.2 \mathrm{M}_{\odot}$. However, as shown in Stasińska et al. it is fairly independent of the PN mass value.

The PNn masses can also be determined from $\log g$ and $T_{\text {eff }}$ derived from model atmosphere analyses of PNn spectra. The resultant mass distribution for $69 \mathrm{PNn}$ which have been investigated in this way is presented in Fig. 2. The data have been taken from Méndez et al. (1992), McCarthy et al. (1997), Rauch et al. (1999), and Napiwotzki (1999). Positions of the objects in the $\log g-\log T_{\text {eff }}$ plane can be found in Schmidt \& Tylenda in these proceedings.

The median mass for the distribution in Fig. 2 is $0.58 \mathrm{M}_{\odot}$. The mean mass is $0.60 \mathrm{M}_{\odot}$ while the standard deviation is $0.096 \mathrm{M}_{\odot}$. These numbers are close to those quoted above and derived from statistical evolutionary considerations. However, the shape of the histrogram in Fig. 2 is rather unexpected. The distribution is bi-modal with a clear minimum around $0.60 \mathrm{M}_{\odot}$. It is difficult to understand why objects with masses close to the value usually considered as typical or canonical have avoided spectroscopic studies. As can be seen from the diagram in Schmidt \& Tylenda (these proceedings) the more massive part of the distribution in Fig. $2\left(\gtrsim 0.60 \mathrm{M}_{\odot}\right)$ is populated by low gravity, cool $\mathrm{PNn}$, i.e. stars evolving horizontally in the HR diagram. In the low mass part 


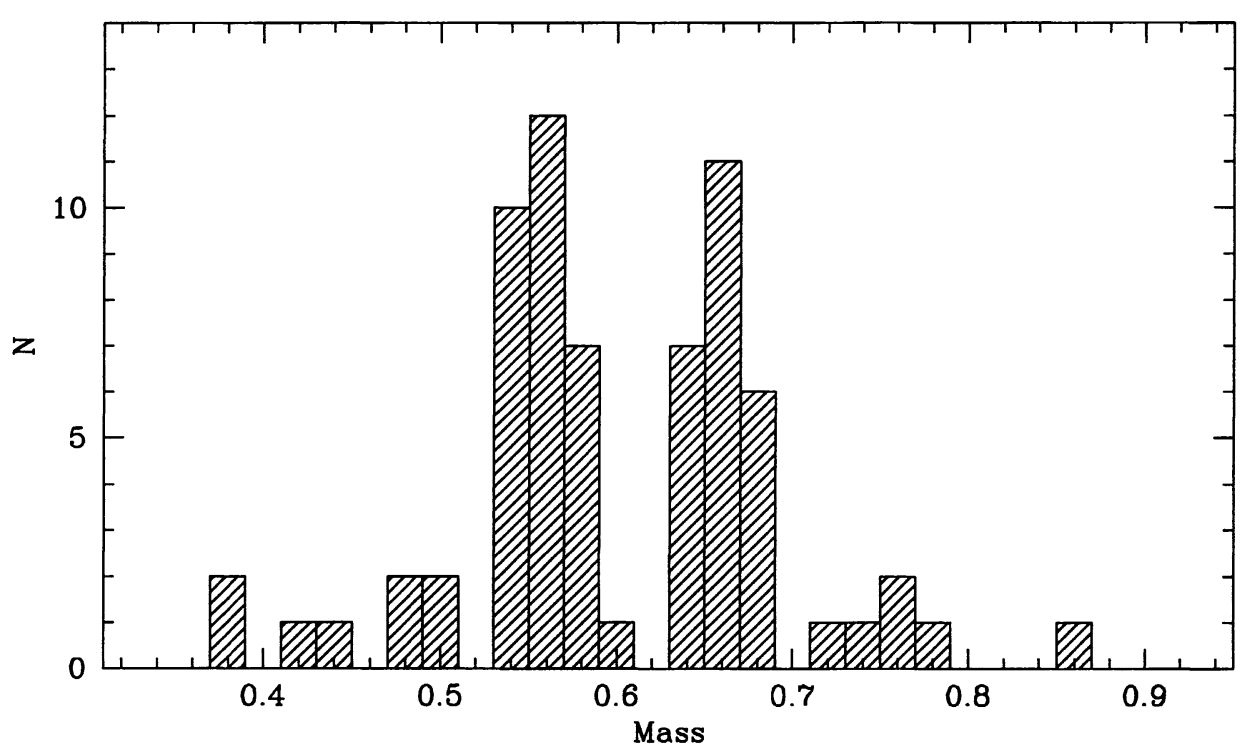

Figure 2. The distribution of the PNn masses (in solar masses) derived from spectroscopic studies (see text for references).

( $\lesssim 0.60 \mathrm{M}_{\odot}$ ), however, mainly PNn in the cooling phase are observed. A straitforward interpretation of the diagram in Schmidt \& Tylenda could be as follows. Low mass stars $\left(\lesssim 0.65 \mathrm{M}_{\odot}\right)$ evolve slowly in the post-AGB phase, increase very significantly their speed while becoming cool, low gravity PNn (thus avoiding observations) and slow down very efficiently in the cooling phase. On the contrary, high mass stars $\left(\gtrsim 0.65 \mathrm{M}_{\odot}\right)$ evolve quickly across the post-AGB and slow down in the PNn phase so much that their PNe disperse in interstellar medium before they become hot, high gravity stars. The parts concerning the PNn phase in the above two sentences are in complete contradiction to what we can learn from the theory of stellar evolution. Thus there is something wrong either with the theory of stellar evolution or with PNn atmosphere models. It is difficult to find any other reasonable explanation.

\section{Evolutionary status of [WC] central stars}

About $10 \%$ of the PNe possess H-deficient central stars, mostly classified as [WC] type or PG1159 type. For reviews of observed characteristics of these stars see Hamann and De Marco et al. in these proceedings. The H-deficient PNn present a certain challenge to the theory of stellar evolution as standard models have not been able to predict their origin. All the standard PNn models keep a H-rich envelope throughout their evolution up to the white dwarf stage.

The [WC] PNn burn helium. Thus their origin must be related to thermal pulses and the following He-burning. The question is when does this He-shell flash takes place. From this point of view three cases can be considered. First, the last AGB pulse which ends the AGB phase and the PN formation. Second, 
a late He-shell flash which happens during the PNn phase when the star evolves horizontally in the HR diagram. Third, a very late He-shell flash which occurs on the cooling part of the PNn track. From theoretical point of view the third possibility seems to be most appealing because the amount of hydrogen to be burnt and/or lost is smallest in this case.

The problem of the evolutionary status of the H-deficient PNn has recently been studied in Górny \& Tylenda (2000). They have found that the very late flash scenario is not compatible with the observational data for the [WC] $\mathrm{PNe}$. Perhaps the most straighforward evidence comes from the distribution of the PN surface brightness. The observed distribution for the $\mathrm{PNe}$ with $\mathrm{H}$-deficient $\mathrm{PNn}$ is the same as for the rest of the PN population. The very late flash scenario predicts a significant difference in the sense that the H-deficient PNn should not be observed amongst the PNe of highest surface brightness. It is important to note that the observational parameters for the PNe surrounding FG Sge and V4334 Sgr are compatible with the late or very late flash scenario. These objects, however, are not evolving towards the parameter region where most of the [WC] PNe are observed. The conclusion of Górny \& Tylenda is therefore that most of the H-deficient PNn, at least of [WC] type, evolve directly from AGB, i.e. they have been formed in the last AGB thermal pulse.

As the youngest [WC] $\mathrm{PNn}$ have atmospheres mostly composed of $\mathrm{He}, \mathrm{C}$ and $\mathrm{O}$, there are two important consequences of the above conclusion. First, the star has to get rid of hydrogen during the post-AGB phase, i.e. before the PN becomes ionized and visible. This can be done by intense mass loss and/or mixing and burning. Second, convective overshooting during the thermal pulse has to be important. Theoretical discussion of these processes can be found in Blöcker and Herwig in these proceedings.

\section{Traces of the PNn evolution in $\mathbf{P N}$ haloes}

Many PNe possess outer faint, extended structures or haloes. Observational studies of these structures are important as these regions can say us about some aspects of the PN and PNn evolution. Low densities in the haloes mean long relaxation times for ionization processes. These times are of order of recombination time for hydrogen which is $\sim 10^{5} / N_{e}$ years (where $N_{e}$ is the electron density in $\mathrm{cm}^{-3}$ ). Thus in PN haloes where $N_{e}$ can be as low as $10 \mathrm{~cm}^{-3}$ the physical state of the nebular material can preserve certain imprints of the past evolution during last $10^{4}$ years or so. Below two cases of non-steady photoionization effects in the PN haloes are discussed.

\subsection{Hot haloes}

It has been known from spectroscopic studies that outer haloes of some PNe are hotter than the inner nebulae (e.g. Middlemass et al. 1989, 1991; Hyung et al. 2001). As steady-state photoionization models cannot account for the observed values usually this fact is interpreted in terms of shock heating (e.g. Middlemass et al. 1991; Hyung et al. 2001). There is, however, another possibility suggested in Marten (1993) which, however, has not been followed in later studies.

One of the main aspects of the PN evolution is the propagation of the ionization front across the nebula. This can be due to the increasing effective 
temperature of the PNn and the resulting increase of the ionizing flux, as well as, the expansion of the PN. In a dense PN the propagation of the ionization front is slower than photoionization processes and the state of the nebular gas remains close to steady-state conditions. However, if the PN has a thin outer halo and the ionization front breaks through the main dense nebula the halo becomes exposed to a strong ionizing flux. The ionization front accelerates and becomes strongly supersonic, i.e. turns into a weak $\mathrm{R}$-front. The principal result is a strong heating of the halo material to a temperature well above the equilibrium value. Because of long cooling time (low density) the halo can remain hot for a long time.

Theoretical modelling of the above scenario can be found in Marten (1993) and in Tylenda (in this volume). It shows that just after the transition of ionization front the electron temperature in the halo can reach values over $210^{4} \mathrm{~K}$. As can be seen from the figure in Tylenda (these proceedings) even 1000 years later the halo as observed in the [OIII] lines would appear significantly hotter than the inner dense nebula.

It is not clear, at present, which mechanism works in real objects. It requires futher observational studies and theoretical works. It should, however, be noted that an ionization front transition has to occur at least once in the history of a PN halo and it should produce effects similar to those in the models. If proved that these effects are really observed in the PNe they could be used for observational measuring of the PNn evolutionary speed.

\subsection{Recombination haloes}

It has been shown in Tylenda (1986) that some PN haloes may result from a fast decline in the PNn luminosity. This decline has been predicted for H-burning PNn when they cease nuclear reactions (e.g. Schönberner 1981). A drop of the ionizing flux by factor 10 provokes recombination of the outer PN regions and their transformation into a faint, low-excitation halo. Recent observational studies of some PNe from those discussed in Tylenda (1986) show that we probably do observe recombination haloes.

One of these objects is NGC 2438 which has been thouroughly studied by Corradi et al. (2000). From their image and spectroscopic observations, as well as, hydrodynamical modelling Corradi et al. conclude that the inner component of the NGC 2438 halo (halo 1 in their notation) is of recombination origin. The outer much fainter component would be the ionized AGB wind.

Very recently, Turatto et al. (2001) have investigated the ionization structure of NGC 6565. The authors have obtained long-slit spectra at different position angles and analysed them together with HST images of the PN. The study shows that faint outer regions are of much lower excitation than the inner bright nebula. Detailed comparison between a model ionization structure and the observations leads the authors to a conclusion that the faint, low-excitation halo of NGC 6565 is of recombination origin. The luminosity decline of the PNn would have started about 1000 years ago.

Observational detections and studies of recombination haloes in PNe are important from the point of view of the PNn evolution theory. They could provide an observational evidence that at least some of the PNn do suffer from 
a fast luminosity decline. These would imply that these PNn have been burning hydrogen when forming their PNe.

\section{Chemical inhomogeneities in the nebulae}

It has been known for long time that the electron temperature determined from the Balmer jump sometimes appears to be significantly lower than that from optical forbidden line ratios (e.g. Peimbert 1971; Liu \& Danziger 1993). Also for a long time it has been found that the CNO abundances from optical recombination lines are sometimes significantly higher than those from optical and UV collisionally excited lines (e.g. Kaler 1986; Barker 1991). Recent spectroscopic studies cast new light on these two puzzling problems and show that they are linked up (Liu et al. 2000, 2001; see also Liu, these proceedings).

These observational facts can be consistently explained assuming that in the PNe there are strong inhomogeneities in chemical abundances. Regions with more or less standard abundances have also standard electron temperatures, i.e. close to $10^{4} \mathrm{~K}$. Regions with $\mathrm{CNO}$ and Ne siginificantly enhanced have much lower temperatures due to enhanced cooling in infrared lines of these elements. In the optical spectrum the cool regions contribute only to recombination lines and continuum but not to collisionally excited (forbidden) lines (because of too a low temperature). Thus an observer analysing only collisionally excited lines in optical and UV analyses only the regions with normal abundances. While analysing recombination lines and Balmer jump he gets results representing an average over the whole nebula.

As shown in Tylenda (these proceedings, see also Péquignot et al., these proceedings) the observational data for NGC 6153, M 2-36 and M 1-42, which are PNe showing extreme effects of this kind, can be accounted for by photoionization model nebulae in which $\sim 1 \%$ of the nebular mass is in form of strongly enriched inclusions. The CNO and Ne enrichment in the inclusions is $\sim 200$. However, the global enrichment in the whole nebula is only $\sim 2$, i.e. much lower than found from standard analyses of the recombination lines (Liu et al. 2000, 2001)

An obvious question that arises is what is the origin of these strongly enriched inclusions. Usual interpretation of observed enhancements in PNe is that they are due to nuclear synthesis in stellar interiors and dredge-up processes, mostly due to the third dredge-up during the thermally pulsating AGB (see Herwig or Lattanzio, these proceedings). If this idea is followed than the enriched inclusions would imply significant confinement of the dredge-up to small stellar regions, at least near the end of AGB when the PN is formed. This seems to be supported by recent hydrodynamic simulations of convection in red giants which show that convective motions are in form of well defined up-streams and down-streams (see e.g. results of Freytag at www.astro.uu.se/ bf). Also magnetic fields, whose important strength can be inferred from maser observations of AGB stars (e.g. Szymczak et al. 1998, 2001), can play important role in confinement of convection and dredge-up. Strongly enriched material appearing in localized regions of the stellar atmosphere whould then be blown away by the AGB wind and would give origin to enriched regions observed in some PNe. 
Acknowledgments. This paper has partly been supported from the Polish State Committee for Scientific Research grant no. 2.P03D.020.17.

\section{References}

Acker, A., Ochsenbein, F., Stenholm, B., Tylenda, R., Marcout, J., \& Schohn, C. 1992, Strasbourg-ESO Catalogue of Galactic Planetary Nebulae, ESO

Barker, T. 1991, ApJ, 371, 217

Bensby, T., \& Lundström, I. 2001, A\&A, 374, 599

Corradi, R. L. M., Schönberner, D., Steffen. M., \& Perinotto, M. 2000, A\&A, 354, 1071 Gathier, R. 1987, A\&AS, 71, 245

Górny, S. K., Stasińska, G., \& Tylenda, R. 1997, A\&A, 318, 256

Górny, S. K., \& Tylenda, R. 2000, A\&A, 362, 1008

Hyung, S., Mellema, G., Lee, S-J., \& Kim, H. 2001, A\&A, 378, 587

Kaler, J. B. 1986, ApJ, 308, 337

Liu, X.-W., \& Danziger, I. J. 1993, MNRAS, 263, 256

Liu, X.-W., Storey, P. J., Barlow, M. J., Danziger, I. J., Cohen, M., Bryce, M. 2000, MNRAS, 312,585

Liu, X.-W., Luo, S.-G., Barlow, M. J., Danziger, I. J., Storey, P. J. 2001, MNRAS, 327, 141

Marten, H. 1993, A\&A, 277, L9

Marten, H., \& Schönberner, D. 1991, A\&A, 248, 590

McCarthy, J.K., Méndez, R.H., \& Kudritzki, R.-P. 1997, in IAU Symp. 180, Planetary Nebulae, ed. Habing \& Lamers, (Dordrecht: Kluwer), 120

Middlemass, D., Clegg, R. E. S., \& Walsh, J.R. 1989, MNRAS, 239, 1

Middlemass, D., Clegg, R. E. S., Walsh, J.R., \& Harrington, J. P. 1991, MNRAS, 251, 284

Méndez, R., Kudritzki, R.P., \& Herrero, A. 1992, A\&A, 260, 329

Napiwotzki, R. 1999, A\&A, 350, 101

Peimbert, M. 1971, Bol. Obs. Tonantzintla y Tacubaya, 6, 29

Pottasch, S. R. 1992, A\&AR, 4, 315

Pottasch, S. R., \& Acker, A. 1989, A\&A, 221, 123

Rauch, T., Köppen, J., Napiwotzki, R., \& Werner, K. 1999, A\&A, 347, 169

Schönberner, D. 1981, A\&A, 103, 119

Schmidt-Voigt, M., \& Köppen, J. 1987, A\&A, 174, 211

Stasińska, G., Górny, S. K., \& Tylenda, R. 1997, A\&A, 327, 736

Stasińska, G., Tylenda, R., Acker, A., \& Stenholm, B. 1991, A\&A, 247, 173

Szymczak, M., Cohen, R. J., \& Richards, A. M. S. 1998, MNRAS, 297, 1151

Szymczak, M., Cohen, R. J., \& Richards, A. M. S. 2001, A\&A, 371, 1012

Turatto, M., Cappellaro, E., Ragazzoni, R., Benetti, S., \& Sabbadin, F. 2001, A\&A, submitted

Tylenda, R. 1986, A\&A, 156, 217

Tylenda, R., \& Stasińska, G. 1989, A\&A, 217, 209

Zhang, C. Y. \& Kwok, S. 1993, ApJS, 88, 137 